\title{
Liderazgo transformacional y clima organizacional en docentes de la Universidad Nacional Intercultural de la Amazonia
}

\section{Transformational leadership and organizational climate in teachers from the Universidad Nacional Intercultural de la Amazonia}

\author{
Dulio Oseda Gago a, , Abdías Chávez Epiquén ${ }^{\text {a }}$,Willian G. Castro Paniagua a \\ a Universidad Nacional Intercultural de la Amazonia, Pucallpa, Perú
}

\section{RESUMEN}

El objetivo fue determinar la relación que existe entre el liderazgo transformacional y el clima organizacional en los docentes de la Universidad Nacional Intercultural de la Amazonia, Pucallpa en el año 2015. La hipótesis general sustenta que existe relación directa y significativa entre el liderazgo y el clima organizacional en los docentes de la Universidad Nacional Intercultural de la Amazonia. La investigación fue de tipo básica y nivel correlacional, método descriptivo e hipotético deductivo, con un diseño descriptivo - correlacional, la muestra censal fue de 114 docentes. La recolección de datos se realizó con la técnica de encuesta y como instrumento se utilizó un cuestionario, asimismo se confiabilizó con el método de mitades partidas de Rulon y Guttman siendo este igual a 0,993 y 0,977 ; la validez se hizo mediante el juicio de expertos y el alfa de Cronbach obteniéndose valores de 0,996 y 0,928. Se concluye que con un nivel de significancia del $5 \%$, existe una relación directa fuerte ( $r h o=0,817)$ y significativa $(t=13,14)$ entre el liderazgo transformacional y el clima organizacional en los docentes de la Universidad Nacional Intercultural de la Amazonia de Pucallpa. Asimismo al realizar el cruce de todas las dimensiones con todas las dimensiones, se apreció que el coeficiente de correlación en todos los casos es directa y significativa en los docentes de la Universidad Nacional Intercultural de la Amazonia, Pucallpa al 2015.

Palabras clave: Liderazgo transformacional, clima organizacional, comunicación, condiciones laborales, involucramiento laboral, autorrealización.

\begin{abstract}
The objective was to determine the relationship between transformational leadership and organizational climate in professors of the National Intercultural University of the Amazonia, Pucallpa in 2015. The general hypothesis holds that there is a direct and significant relationship between leadership and organizational climate in professors from the National Intercultural University of the Amazonia. The research was basic and correlational level, descriptive and hypothetical deductive method with a design descriptive - correlational, the census sample was 114 professors. Data collection was performed using the survey technique as an instrument was used a questionnaire, also it was validated with the method of split halves of Rulon and Guttman, this being equal to 0.993 and 0.977 ; the validity judgment was made by experts and Cronbach's alpha values obtained 0.996 and 0.928 . We concluded that with a significance level of $5 \%$, there is a strong direct relationship (rho $=0.817$ ) and significant $(t$ $=13.14$ ) between transformational leadership and organizational climate in professors of the National Intercultural University of the Amazonia of Pucallpa. In addition to performing all dimensions crossing all dimensions, it was noted that the correlation coefficient in all cases is direct and meaningful in teachers participation from the National Intercultural University of the Amazonia, Pucallpa 2015.
\end{abstract}

Keyword: Transformational leadership, organizational climate, communication, working conditions, job involvement, self-realization.

Historial del artículo:

Recibido, 25 de enero de 2016; aceptado, 13 de marzo de 2016; disponible en línea, 30 de marzo de 2016

* Lic. en Educación Secundaria, Mg. en Docencia en Educación Superior, Dr. en Ciencias de la Educación, Postdoctorado en Sistemas Diacrónicos y Sincrónicos de la Investigación Científica. Profesor Principal de la Universidad Nacional Intercultural de la Amazonía.

Correo: dosedag@hotmail.com

Este es un artículo de acceso abierto, licencia CC BY (https://creativecommons.org/licenses/by/4.0/) 


\section{INTRODUCCIÓN}

Habiendo realizado el diagnóstico de la realidad problemática podemos enfocarla desde una perspectiva del contexto internacional, según Donayre "los problemas referidos al clima organizacional son tan comunes, principalmente en las organizaciones educativas públicas" (1). Si bien es cierto la administración educativa está presente en todas las organizaciones educativas, la misma que facilita el cumplimiento de los objetivos educativos, también es necesario contar con la parte pedagógica y clima organizacional.

Asimismo a nivel nacional, según la reforma del currículo por competencias que impulsa el Ministerio de Educación dentro de sus políticas ha priorizado las condiciones del clima en aula y en la institución educativa, dando énfasis a la gestión centralizada en los aprendizajes donde todo cuerpo directivo, jerárquico y docente de la institución educativa labore en sintonía a la buenas relaciones humanas.

Estos cambios ocasionan desajustes especialmente en las actuales autoridades educativas de las instituciones educativas a nivel nacional, como es el caso de la Universidad Nacional Intercultural de la Amazonia.

En los últimos años la región Ucayali está a nivel nacional en los últimos lugares en las evaluaciones ECE (último y penúltimo lugar) en el segundo grado de primaria, siendo uno de los factores las deficientes relaciones humanas entre el cuerpo directivo, docente y el acompañante del MINEDU de las instituciones educativas; y este fenómeno se hace también extensivo a nivel secundario y también superior no universitario y universitario. En este contexto el clima organizacional y liderazgo es de suma importancia en el desarrollo institucional, especialmente en la gestión centralizada en los aprendizajes de los estudiantes universitarios.

A nivel local y/o institucional, se ha podido observar que la comunidad universitaria de la Universidad Nacional Intercultural de la Amazonia de Pucallpa, tienen dificultades de manera directa en sus relaciones humanas, ya que como se ha podido apreciar, en casi todos los sectores públicos de nuestro país existen dos grupos bien diferenciados y antagónicos que luchan por el poder, comenzando desde el proceso de planificación, ejecución y evaluación en las entidades públicas.

De otro lado la interacción de los agentes educativos, su forma de organización y administración que se da función a la Ley Universitaria 30220; el cual se manifiesta en su desorganización cuando estos toman sus decisiones al interior, asimismo el ambiente imperante vienen a ser en muchos casos el autoritarismo, y el trato entre el personal directivo
(Presidente y el vicepresidente académico) con los docentes en la mayoría de los casos es horizontal, pero tiende al verticalismo.

Todos estos aspectos también se encuentran detallados y documentados en el Plan Estratégico de la Universidad, recopilados lógicamente mediante el Análisis DAFO, la Técnica del Pert CPM y el diagrama causal de Forest.

Frente a ello el estudio de liderazgo transformacional y el clima organizacional es importante, ya que esta contribuye a superar dichas dificultades que afectan a la Universidad pública. Por lo tanto se formula las siguientes preguntas de investigación; ¿QQué relación existe entre el liderazgo transformacional y el clima organizacional en los docentes de la Universidad Nacional Intercultural de la Amazonia, Pucallpa 2015?.

El objetivo fue determinar la relación que existe entre el liderazgo transformacional y el clima organizacional en los docentes de la Universidad Nacional Intercultural de la Amazonia, Pucallpa - 2015.

La hipótesis general formulada: Existe relación directa y significativa entre el liderazgo y el clima organizacional en los docentes de la Universidad Nacional Intercultural de la Amazonia, Pucallpa 2015.

Respecto al marco teórico; según Alvarez (2007) el vocablo "líder" probablemente sea uno de los más revisados por la literatura contemporánea. Basta con detenerse a echar una mirada a revistas, periódicos, libros y publicaciones para comprobar hasta qué punto se ha extendido y generalizado su uso. Esto ha provocado, mucha ambigüedad en el uso del término, que se emplea indistintamente. Se logra entender lo mismo cuando calificamos como líder a un dirigente político, empresarial o religioso. Según Lazzati (2011), "El liderazgo es la actividad de influenciar en la gente para que se empeñe voluntariamente en el logro de los objetivos del grupo. Por grupo debe entenderse un grupo pequeño, un sector de la organización, una organización, una nación, etc." (2).

Respecto al liderazgo transformacional surge a partir de Mc Gregor Burns y Bernad Bass, que describieron una serie de cualidades que conformaban un tipo de liderazgo empresarial que se comenzaba a valorar más en el mundo de la empresa. Se trataba del concepto de liderazgo transformacional. En si el proceso común de líder y trabajadores para avanzar a un nivel más alto de la moral y la motivación. Es una transformación que produce cambios significativos en la empresa y en las personas que la conforman.

Por lo tanto el liderazgo transformacional, es definido 
como el proceso de influir, guiar y dirigir a los miembros de un grupo u organización, quienes siguen al líder de forma voluntaria en la búsqueda del éxito en la consecución de unos objetivos. Se han realizado extensos estudios sobre la materia y los investigadores han diferenciado varios tipos de liderazgo, siendo el transformacional uno de los que más atención ha recibido.

El concepto de liderazgo transformacional fue originado e introducido por el experto en liderazgo Bass (1997), este lo definió como el tipo de liderazgo ostentado por aquellos individuos con una fuerte visión y personalidad, gracias a la cual son capaces de cambiar las expectativas, percepciones y motivaciones, así como liderar el cambio dentro de una organización. Asimismo, determinó que tal tipología de liderazgo era observable "cuando los líderes y seguidores trabajan juntos para avanzar a un nivel superior de moral y motivación" (3).

Las dimensiones del Liderazgo Transformacional, a continuación se desarrollan las dimensiones del Liderazgo Transformacional. Según Bass (1997) el líder transformacional se distingue por cuatro características básicas, relacionándolas con la labor desempeñada por el docente (3): Estimulación intelectual, motivación inspiracional, consideración individualizada e influencia idealizada.

Ahora vamos a documentar la segunda variable de estudio, el clima organizacional es el nombre dado por diversos autores; al ambiente generado por las emociones de los miembros de un grupo u organización, el cual está relacionado con la motivación de los empleados. Se refiere tanto a la parte física como emocional. El concepto se asimila al de dinámica de grupo al analizar las fuerzas internas que inciden en el ambiente laboral como resistencia al cambio. Fue el sociólogo Kurt Lewin quien desarrolló un análisis del campo de fuerzas, como modelo con el cual describía cualquier nivel presente de rendimiento. Algunos autores consideran al clima organizacional como las percepciones colectivas e individuales que tienen los trabajadores sobre su organización, influenciadas por variables psicosociales, laborales y organizacionales, que repercuten sobre el comportamiento organizacional y la productividad empresarial.

De esta definición se puede afirmar que el clima organizacional es un concepto que está referido a la vida organizacional en una empresa. Es así, que el clima organizacional es configurado como una dimensión o ámbito evaluativo básico y es definido como: "La calidad del entorno global de una organización (4)".

Para Alvarado (2003) el Clima Organizacional comprende la suma de las percepciones que describe el grado de satisfacción de las personas, de su medio laboral en que se desarrolla el trabajo cotidiano. Corroborando lo mencionado se reafirma, que es una percepción que se tiene de la persona y del medio ambiente laboral y consiste en el grado favorable o desfavorable del entorno laboral para las personas que integran la organización (5).

La existencia de algunas categorías o dimensiones existentes del clima organizacional en una organización se relacionan con propiedades propias de cada uno. Es necesario precisar que dichas categorías no son únicas, como tampoco existe un clima único o ideal depende de las características propias de cada entidad. Según Rodríguez (1999), la comunicación, las condiciones laborales, el involucramiento laboral, la autorrealización y la supervisión (6).

\section{MATERIAL Y MÉTODOS}

Se utilizó el diseño descriptivo correlacional. El esquema es el siguiente:

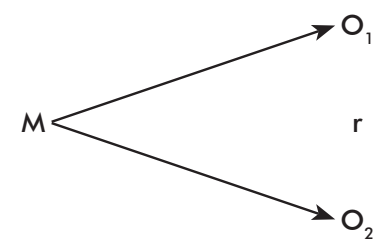

Donde:

$M \quad=$ Muestra

O1 = Observación de la variable 1 .

O2 = Observación de la variable 2 .

$r \quad=$ Correlación entre dichas variables.

En el caso de nuestra investigación, la población estuvo conformada por 114 docentes de la Universidad Nacional Intercultural de la Amazonia, Pucallpa 2015. El Muestreo fue probabilístico, y conformado por 88 docentes.

Respecto a las técnicas de recolección de datos, se aplicó una encuesta con su instrumento el cuestionario. La confiabilidad se hizo por el método de mitades partidas y la validez por el juicio de experto.

\section{RESULTADOS}

A continuación se muestra el consolidado de las variables "Liderazgo transformacional" y "Clima organizacional", el cual se aplicó a la muestra de 88 docentes de la Universidad Nacional Intercultural de la Amazonia, Pucallpa-2015. 
Según la tabla 1 se puede apreciar que los niveles de la variable 1, Liderazgo transformacional de los 88 docentes encuestados, 50 docentes que representa el $(56,86 \%)$ perciben que el liderazgo transformacional es bueno; luego 34 docentes que representan el $(38,64$ $\%)$ perciben el liderazgo transformacional como muy bueno; luego 4 docentes que es el (4,55 \%) perciben

Tabla $N^{\circ}$ 1: Niveles de la variable 1: Liderazgo Transformacional.

\begin{tabular}{lcc}
\hline \multicolumn{1}{c}{ Niveles } & Niveles & Niveles \\
\hline Muy deficiente & 0 & 0,00 \\
Deficiente & 4 & 4,55 \\
Bueno & 50 & 56,86 \\
Muy bueno & 34 & 38,64 \\
Total & 88 & 100,00 \\
\hline Fuente: Bases de datos & Liderazgo & Transformacional, \\
\multicolumn{1}{c}{ 1999). } & &
\end{tabular}

el liderazgo transformacional como deficiente. En el nivel muy deficiente no se tuvo a ningún docente de la Universidad.

Respecto a la segunda variable (tabla 2) se puede apreciar que los niveles de la variable 2 "Clima organizacional" de los 88 docentes encuestados, 49 docentes que representan el $(55,68 \%)$ perciben un clima organizacional favorable; luego 25 docentes que representan el $(28,41 \%)$ perciben un clima

Tabla $N^{\circ}$ 2: Niveles de la variable 2: Clima Organizacional.

\begin{tabular}{lcr}
\hline \multicolumn{1}{c}{ Niveles } & Niveles & Niveles \\
\hline Muy desfavorable & 0 & 0.00 \\
Desfavorable & 14 & 15.91 \\
Favorable & 49 & 55.68 \\
Muy favorable & 25 & 28.41 \\
Total & 88 & 100.00 \\
\hline
\end{tabular}

Fuente: Base de datos Clima organizacional.

organizacional favorable; luego 14 docentes que son el $(15,91 \%)$ perciben un clima organizacional desfavorable y ningún docente percibe que el clima organizacional sea muy desfavorable.

Respecto a la prueba de hipótesis, como el diseño utilizado fue el descriptivo - correlacional, el estadígrafo utilizado fue el coeficiente de correlación de Spearman, prueba bilateral o a dos colas. Ahora bien, como la rho=0,817 y la † calculada (tc) con los datos procesados es igual a: 13,14 y este cae en la zona de rechazo; entonces se rechaza la Ho, y se acepta la hipótesis alterna $\mathrm{H} 1$; que dice: Se concluye que existe una relación directa y significativa entre el liderazgo transformacional y el clima organizacional en los docentes de la Universidad Nacional Intercultural de la Amazonia, Pucallpa - 2015.

\section{DISCUSIÓN}

Según la aplicación de los dos cuestionarios en base a la percepción docente sobre el liderazgo transformacional y el clima organizacional en los docentes de la Universidad Nacional Intercultural de la Amazonia, Pucallpa - 2015.

Contrastando el problema o dificultad que presentan la mayoría de instituciones educativas, según la muestra de estudio que es la Universidad Nacional Intercultural de la Amazonia con los problemas o dificultades que se han investigado en otras realidades, para encontrar si es que existen semejanzas o diferencias, se encontró en relación con la variable 1, Liderazgo transformacional los resultados son los siguientes: un gran porcentaje percibe un nivel bueno $(56,82 \%)$, muy bueno $(38,64$ $\%)$ y deficiente el $(4,55 \%)$.

En consecuencia de ello se afirma que el liderazgo transformacional en la Universidad Nacional Intercultural de la Amazonia es bueno y/o aceptable en la percepción de los docentes, pero se debe tener en cuenta la percepción de aquellos docentes que no la consideran favorable.

Estos resultados refuerzan lo encontrado en los antecedentes de la tesis de Ruiz (2011) cuando concluye que el estilo de liderazgo del director (Rector - Presidente de la Comisión de Gobierno) influye significativamente en la eficacia de las instituciones educativas del Consorcio "Santo Domingo de Guzmán" de Lima Norte. (8)

En los datos obtenidos en la variable 2, Clima organizacional se evidencia en mayor porcentaje en el nivel favorable siendo este el 55,68 \% y muy favorable $28,41 \%$ respectivamente en la percepción de los mismos docentes y en los niveles desfavorable 15,91 $\%$ y muy desfavorable 0,00 \%.

Como consecuencia de ello se afirma que el clima organizacional en la Universidad Nacional Intercultural de la Amazonia es aceptable en la percepción de los docentes, pero se debe tener en cuenta la percepción de aquellos docentes que no la consideran favorable, aunque es un pequeño grupo, peor debe tenerse en cuenta.

Estos resultados son corroborados con los encontrados en los antecedentes en la tesis de Montalvo (2008) denominado: Relación entre clima organizacional y el desempeño docente donde el $51 \%$ de los sujetos 
perciben el clima organizacional en un alto nivel, muy similar a los encontrados en la presente investigación (9).

Respecto a la hipótesis general y estadística de investigación dice literalmente: Existe una relación directa y significativa entre el liderazgo transformacional y el clima organizacional en los docentes de la Universidad Nacional Intercultural de la Amazonia, Pucallpa - 2015. En el coeficiente de correlación entre la variable liderazgo transformacional y el clima organizacional, la "rho" de Spearman $=0,817 \mathrm{con}$ el nivel de significancia de $5 \%$ este es considerado como correlación positiva fuerte y en la decisión estadística se evidencia que la tc es mayor que $\dagger$ teórica $(13,14>1,66)$, en consecuencia se rechaza la hipótesis nula $(\mathrm{Ho})$ y se acepta la hipótesis alterna $(\mathrm{Hi})$. Se concluye que existe una correlación directa fuerte y significativa entre el liderazgo transformacional y el clima organizacional en los docentes de la Universidad Nacional Intercultural de la Amazonia, Pucallpa 2015.

Dichos resultados son corroborados en los encontrados en los antecedentes de la tesis de Montalvo (2008) cuando concluye que existe una relación entre el clima organizacional y el nivel de desempeño docente, que es percibido por el docente y los estudiantes; el $51 \%$ de los sujetos encuestados percibe el clima organizacional en un alto nivel, así como el $64 \%$ de los entrevistados percibe el desempeño docente en el nivel alto respectivamente y efectuar la correlación entre el liderazgo de los directores y la planificación de la enseñanza, se demuestra que existe una correlación moderada, la relación se expresa en un 65 \% (9).

Asimismo se ha visto que en las últimas décadas se ha mencionado mucho como el liderazgo ejerce influencia sobre sus subordinados. Esta es una condición humana básica y global, pues desde los inicios del ser humano donde ya vivían en sociedad han existido líderes que han guiado a los demás, de tal manera que a medida que la sociedad ha ido evolucionando, el liderazgo también se ha transformado (Barroso, 2010) (10).

Podríamos asumir que el comportamiento del líder influye en sus seguidores, siendo el principal responsable del éxito o fracaso de ellos, que son los encargados de ayudar al cumplimiento de los objetivos organizacionales (Morán, 2007) "expresado de otra manera el líder tiene en su poder el progreso de las organizaciones, ya que todas necesitan de alguien para ser dirigidas" (11).

Asimismo para Ponce, (2014) "el clima organizacional influye directamente en el proceso y desarrollo de las funciones de las organizaciones, es decir la percepción que tiene el talento humano del lugar en el que se desempeña que influye en su actuar" (12).
Estas percepciones son las que le dan vida al clima organizacional y la gestión de la dirección cumple el papel más importante para le generación de un clima desfavorable o favorable, por eso se dice que la existencia real de un clima está dada por la subjetividad de las personas que laboran en una organización.

Respecto al liderazgo transformacional es uno de los factores más determinantes e influyentes en la percepción que tienen los trabajadores del clima organizacional (Ponce, 2014) y dada la importancia que tiene el clima organizacional en el cumplimiento de los objetivos de la organización, un clima positivo genera un mayor sentimiento de pertinencia hacia la organización provocando automáticamente un mejor desempeño en los trabajadores (12).

Para Peraza (2004) "por el contrario, un clima negativo disminuye el desempeño ocasionando bajo rendimiento y situaciones de conflicto" (13). La presente investigación considera una revisión teórica del liderazgo, sus antecedentes, conceptos y teorías y a través del análisis de estudios previos determinar su relación sobre el clima organizacional.

Ahora veamos el análisis en las dimensiones, comencemos por la primera. La primera dimensión estimulación intelectual del Liderazgo transformacional el $(54,55 \%)$ perciben que la estimulación intelectual es buena; el $(34,09 \%)$ perciben que la estimulación intelectual es muy buena; luego el (11,36 \%) perciben una estimulación intelectual deficiente el cual es preocupante.

De esta manera se puede afirmar que la estimulación intelectual es en terminos generales buena y/o aceptable en la percepción de los docentes de la Universidad Nacional Intercultural de la Amazonia, Pucallpa - 2015. Esto es corroborado por Bass (1997) cuando menciona que el líder promueve nuevos enfoques y nuevas soluciones a los problemas. A la vez, provoca intelectualmente a su gente estimulándoles a hacerse preguntas, cuestionando los modos habituales de hacer las cosas, permitiendo que tengan errores.

Según Aguilera (2011) reafirmamos la conclusión en términos mayoritarios que lo Centros Educativos se identifican con tipos de liderazgos bastante positivos con unos niveles de satisfacción bastante altos de los líderes hacia los docentes y viceversa (14). Se valora de forma muy positiva y es mencionado en muchos caso el esfuerzo extra que realizan en la institución todos sus empleados. También la efectividad de estos, y en general se rechazan aquellos tipos de liderazgo que implican el acto de eludir responsabilidad por parte del líder y la pasividad ante las tomas de decisiones realizadas.

La segunda dimensión motivación inspiracional del 
Liderazgo transformacional el (48,86\%) perciben que la motivación inspiracional es buena; el $(40,91$ $\%)$ perciben que la motivación inspiracional es muy bueno; luego el (10,23\%) perciben una motivación inspiracional deficiente.

Por ello se afirma que la motivación inspiracional es aceptable en la percepción de los docentes de la Universidad Nacional Intercultural de la Amazonia, Pucallpa - 2015. Esto es corroborado por Bass (1997), citado por (Bernal, 2000) cuando menciona que el líder crea una visión estimulante y atractiva para sus seguidores. Además sabe comunicar su visión de modo convincente con palabras y también con su propio ejemplo (15).

En relación a los resultados evidenciados en los docentes y directores, con respecto al liderazgo transformador desde la perspectiva del líder y como seguidor, se encontró que esta resultó ser media alta con una media dispersión.

Los grandes propósitos de tener un buen liderazgo transformacional para un buen clima organizacional son: incrementar la productividad, disminuir el ausentismo, reducir costos y aumenta sobre manera el desempeño que permita a la organización alcanzar el éxito.

Existen algunas dimensiones que determinan el clima organizacional, sin embargo el liderazgo transformacional se convierte en la más determinante. El estilo de liderazgo referido a la motivación inspiracional que se emplee en la organización influye directamente en el clima organizacional, ya que es este el que logra las percepciones de orgullo de pertenencia, reconocimiento, comunicación y flexibilidad organizativa concordando con (Barroso, 2010); a través de su capacidad para motivar, de generar bienestar general en el trabajador y satisfacción por el trabajo. En pocas palabras es quien logra la percepción de los trabajadores sobre el clima organizacional (10).

Un desempeño de excelencia del trabajador se puede lograr a través de una gran motivación, la misma que es generada por un clima organizacional favorable y este a su vez generado por una buena práctica de liderazgo.

La tercera dimensión consideración individualizada del Liderazgo transformacional, el (61,36 \%) perciben que la consideración individual es buena; el $(25,00$ $\%)$ perciben que la consideración individual es muy buena; luego el (11,36 \%) perciben una consideración individual deficiente.

De ese modo se afirma la consideración individual es buena y/o aceptable en la percepción de los docentes de la Universidad Nacional Intercultural de la Amazonia, Pucallpa - 2015. Esta idea es corroborado por (Bass, 1997) el líder tiene en cuenta las necesidades de cada persona para guiar a cada una según su potencial (3) El liderazgo desde el punto de vista de la consideración individualizada es un concepto cultural, social y sobre todo histórico que ha permitido entender a las organizaciones en distintos contextos como lo confirma (Friedman, 2010) desde la revisión histórica realizada sobre el liderazgo y su incidencia en clima organizacional donde concluye que "el líder es el principal generador de la calidad del clima organizacional debido a su capacidad para formar en los trabajadores aquellas percepciones que le dan vida al clima organizacional" (16).

El líder es quien logra que las organizaciones obtengan los resultados esperados a través de la creación de oportunidades que permitan transformar el contexto lo que corrobora lo expuesto por (Mansilla, 2007) "Es quien influye en los trabajadores, interviniendo en el actuar de estos de tal forma que genera una motivación que permite el máximo desempeño en busca de la excelencia organizacional" (17).

Y la cuarta dimensión influencia idealizada del Liderazgo transformacional el $(64,77 \%)$ perciben que la influencia idealizada es muy buena; el $(32,95 \%)$ perciben que la influencia idealizada es deficiente; el $(2,27 \%)$ perciben una influencia idealizada deficiente. Un líder eficaz es transformador, soportando su comportamiento en valores orientados hacia el bien común tal. Se convierte en exitoso cuando logra el equilibrio entre el cumplimiento de las metas y la preocupación por sus subordinados.

Lo más importante en el líder que influye sobre sus ideales son sus prácticas, ya que para mejorar el desempeño de sus subordinados dependerá exclusivamente en la medida que las prácticas del liderazgo mejoren confirmado por (Cohen, 1974) en base a esto el líder será capaz de fomentar el crecimiento personal y desarrollar habilidades y competencias en los miembros y equipos de la organización. Si el líder no logra que sus seguidores trabajen con entusiasmo y disposición para el logro de sus metas, sencillamente no está siendo eficaz (18).

En virtud de ello se puede definir que el liderazgo como "un proceso de influencia sobre las actividades de individuos o grupos, para lograr metas comunes en situaciones determinadas" (Cantón, 2001) (19).

Algunas conclusiones son:

Se ha determinado con un nivel de significancia del $5 \%$ que existe una relación directa fuerte ("rho" $=0,817$ ) y significativa entre el liderazgo transformacional y el clima organizacional en los docentes de la Universidad 
Nacional Intercultural de la Amazonia, Pucallpa 2015; es decir a mayor liderazgo transformacional, mejor será el clima organizacional de la Universidad.

El nivel de liderazgo transformacional predominante en la percepcion de los docentes de la Universidad Nacional Intercultural de la Amazonia, Pucallpa 2015 , consideran el nivel bueno siendo este el 56,86 $\%$, luego muy bueno el $38,64 \%$ y deficiente el $4,55 \%$.

El nivel de clima organizacional percibido por los docentes de la Universidad Nacional Intercultural de la Amazonia, Pucallpa - 2015, es el favorable en el $55,68 \%$, luego muy favorable en el $28,41 \%$, y finalmente muy desfavorable en el 15,91\%.

\section{REFERENCIAS BIBLIOGRÁFICAS}

1. Donayre W. Administración del Talento Humano. Bogotá: McGraw-Hill; 2009.

2. Lazzati M. Liderazgo de directivos contemporáneos. Lima: Ed. San Marcos; 2011.

3. Bass B. Improving Organizacional Effectives Though Transformational. Leadership. New York: Sage; 1997.

4. Barba D. El clima de trabajo, generado por la cultura organizacional y su influencia como determinante en el comportamiento del personal docente y administrativo de la Escuela Superior de Educación Física de la UAS. [Tesis doctoral]. México: Universidad Autónoma Metropolitana de México; 2011.

5. Alvarado J. Influencia del Liderazgo en la competitividad de las organizaciones peruanas. Lima: PUCP; 2003.

6. Rodriguez D. Liderazgo Gerencial. Bogotá: McGraw-Hill; 1999.

7. Kerlinger $F$, Lee $H$. Investigación del comportamiento. México: Interamericana; 2002.

8. Ruiz P. Influencia del estilo de liderazgo del director en la eficacia de las instituciones educativas del consorcio Santo Domingo de Guzmán de Lima Norte. [Tesis de posgrado]. Lima: Universidad Nacional Mayor de San Marcos; 2011.

9. Montalvo L. El clima organizacional y su influencia en el desempeño docente en las instituciones educativas del nivel de educación secundaria de la UGEL 15 de Huarochirí-2008. [Tesis doctoral]. Lima: Universidad Nacional de Educación; 2008.

10. Barroso F. Liderazgo y clima organizacional en maquiladoras textiles de exportación. México: Interamericana; 2010.

11. Morán A. Liderazgo en la función directiva. México: McGraw-Hill; 2007.

12. Ponce P. Liderazgo femenino y clima organizacional, en un instituto universitario. México: McGraw-Hill; 2014.

13. Peraza Y. Clima organizacional: Conceptos y experiencias. Transporte Desarrollo y Medio Ambiente. México: Interamericana; 2004.

14. Aguilera A. Liderazgo y clima de trabajo en las instituciones educativas de las Fundación Creando Futuro. [Tesis doctoral]. Madrid: Universidad de Alcalá; 2011.

15. Bernal J. Liderar el cambio: El liderazgo transformacional. Madrid: Universidad de Valencia; 2000.

16. Friedman W. Leadership and history. In Nohria N, Khurana R. Handbook of Leadership Theory and Practice. Boston: Harvard; 2010.

17. Mansilla F. Influencia del estilo directivo, el liderazgo estratégico y la Gestión eficaz de tres directores en el rendimiento promedio de los estudiantes de la cohorte educativa 2001 - 2005 en la Institución Inmaculada Concepción. Los Olivos, Lima Perú. [Tesis doctoral]. Lima: Universidad Nacional Mayor de San Marcos; 2007.

18. Cohen M. Leadership and ambiguity: The American College president. New York: McGrawHill; 1974.

19. Cantón L. Gestión de Recursos Humanos. Colombia: McGraw-Hill; 2001. 